\title{
Estimation of left ventricular stroke volume based on pressure waves measured at the wrist: a method aimed at home-based use ${ }^{1}$
}

\author{
Zhipeng Deng ${ }^{\mathrm{a}}$, Chungang Zhang ${ }^{\mathrm{b}}$, Ping $\mathrm{Yu}^{\mathrm{b}}$, Jun Shao ${ }^{\mathrm{c}}$ and Fuyou Liang ${ }^{\mathrm{a}, \mathrm{d}^{*}}$ \\ ${ }^{a}$ School of Naval Architecture, Ocean \& Civil Engineering, Shanghai Jiao Tong University, 800 \\ Dongchuan Road, Shanghai, China \\ ${ }^{b}$ Sky Innovation Technology (Shanghai) Co., Ltd., 2016 Yishan Road, Shanghai, China \\ ${ }^{c}$ The first people's Hospital of Kunshan, 91 West Qianjin Road, Kunshan, Jiangsu, China \\ ${ }^{d}$ SJTU-CU International Cooperative Research Center, Shanghai Jiao Tong University, 800 \\ Dongchuan Road, Shanghai, China
}

\begin{abstract}
Continuous monitoring of stroke volume (SV) or cardiac output (CO) has long been the subject of numerous studies. The majority of existing methods are calibration-dependent, requiring invasive measurements of CO to initialize the estimation algorithms, thus limiting their application to the clinical setting. In the present study, a new calibration-free method aimed at home-based use has been developed, which allows noninvasive estimation of SV from oscillometric signals measured at the wrist. The estimation equation was constructed based on the PRAM method, with significant modifications to incorporate more patient-specific information. Furthermore, the estimation equation was optimized based on the clinical data acquired from 96 patients (the 'Training' group) to obtain the best comparison of estimated SV with echocardiographic SV. The resulting estimation equation was then applied directly to another patient group (the 'Testing' group) to examine its validity. Obtained results demonstrate that our estimations correlated closely with the measurements in both patient groups. In addition to being noninvasive and calibration-free, the proposed method can be fully automated, which may be valuable for the future development of home-based cardiac monitoring systems.
\end{abstract}

Keywords: Stroke volume, oscillometric signals, noninvasive estimation, coefficient optimization

\section{Introduction}

Left ventricular stroke volume (SV) is an important indicator of cardiac function, and a direct measure of cardiac output $(\mathrm{CO})$ if the heart rate is known. For this reason, continuous monitoring of

\footnotetext{
${ }^{1}$ Z.P. Deng performed the numerical analysis and drafted the manuscript; C.G. Zhang and P. Yu collected the clinical data and performed wavelet analysis; J. Shao recruited the patients and performed the clinical measurements; F.Y. Liang designed the study, developed the estimation method and revised the manuscript.

${ }^{*}$ Corresponding author: Fuyou Liang, School of Naval Architecture, Ocean and Civil Engineering, Shanghai Jiao Tong University, 800 Dongchuan Road, Shanghai 200240, China. Tel.: +86 213420 5120; Fax: +86 213420 7804; E-mail: fuyouliang@sjtu.edu.cn.
} 
SV (or CO) is usually required in clinical practice, particularly for patients undergoing surgery or admitted to the intensive care unit [1].

Doppler echocardiography is the method most frequently used to noninvasively determine SV (or $\mathrm{CO})$ in the clinical setting, although its accuracy is only moderate [2,3]. More accurate methods usually require insertion of a catheter into the pulmonary artery, such as the thermodilution method, which has become the gold standard for $\mathrm{CO}$ monitoring in the past decades [4]. The thermodilution method is, however, invasive in nature, carrying with it the potential for serious complications, and for which reason, the frequency of its use is decreasing [5]. In addition, the aforementioned methods do not permit continuous cardiac monitoring. In this context, much effort has been devoted to developing new methods that allow continuous monitoring of $\mathrm{CO}$ without or with little requirement of invasive operations. Arterial pulse wave analysis methods are among those most extensively addressed in recent years [6-9]. The advantage of such approaches lies in that they make use of peripheral arterial pressure waves that can be measured continuously with well-established noninvasive techniques, such as arterial tonometry or finger-cuff photoplethysmography. According to their working principles, existing methods can be classified into two families, namely, the calibration-dependent family [6-8] and the calibration-free family [9]. Despite the emergence of increasing evidence demonstrating the clinical significance of these methods [10-14], none of the methods can be applied in a home-based environment due to the requirement of professional techniques to perform reliable measurements.

Although home-based arterial pressure monitoring has nowadays become very common, it remains unclear whether monitoring ventricular $\mathrm{SV}$ or $\mathrm{CO}$ can provide additional insight into the cardiovascular status of asymptomatic subjects. Theoretically, SV (or CO) may better reflect the working status of the circulation in comparison with arterial pressure since the latter is usually controlled within physiological ranges by the neural and hormonal systems even under pathological conditions. Physiologically, most organs depend on blood flow rather than blood pressure to maintain a normal functional state, which underlines the significance of $\mathrm{CO}$ monitoring. Moreover, from the point of view of pharmacological study, long-term monitoring of $\mathrm{CO}$ is necessary to gather sufficient data to evaluate the effectiveness of pharmacological interventions in improving cardiovascular function [15]. Unfortunately, an approach well-suited to such $\mathrm{CO}$ monitoring does not yet exist according to a recent review study [15].

Recent studies have demonstrated that the wave profile of the oscillometric signals detected in the cuff of an automatic blood pressure device is similar to that of the under-cuff arterial pressure waves when the cuff is operated under certain conditions [16,17]. This raises the possibility of measuring radial pressure waves using an oscillometric blood pressure device and subsequent estimation of SV (or $\mathrm{CO}$ ) using pressure wave analysis in a home-based environment. The purpose of the present study is to develop a method for estimating SV from the oscillometric signals recorded by a blood pressure device mounted at the wrist. For this purpose, we constructed a new SV estimation equation by modifying the equation employed in the pressure recording analytical method (PRAM) [9]. In order to determine the coefficients of the equation, we employed a parameter optimization algorithm to search for the coefficients that result in best matching of estimations with measurements. We divided the patients included in our study into two groups, namely, the 'Training' group and the 'Testing' group. The data of the 'training' group were used to optimize the estimation equation, while the data of the 'testing' group were used to examine the validity of the equation. 
Table 1

Patient information of the 'Training' and 'Testing' groups

\begin{tabular}{lllllll}
\hline $\begin{array}{l}\text { Patient } \\
\text { group }\end{array}$ & $\begin{array}{l}\text { Age } \\
(\text { Years })\end{array}$ & $\begin{array}{l}\text { Sex } \\
(\text { Male/Female })\end{array}$ & $\begin{array}{l}\text { Height } \\
(\mathrm{cm})\end{array}$ & $\begin{array}{l}\text { Weight } \\
(\mathrm{Kg})\end{array}$ & $\begin{array}{l}\text { Syst. Pressure } \\
(\mathrm{mmHg})\end{array}$ & $\begin{array}{l}\text { Dias. Pressure } \\
(\mathrm{mmHg})\end{array}$ \\
\hline Training & $53.4 \pm 14.6$ & $55 / 41$ & $165.9 \pm 8.4$ & $66.8 \pm 11.1$ & $123.6 \pm 12.3$ & $81.0 \pm 11.6$ \\
\hline Testing & $59.0 \pm 14.3$ & $43 / 53$ & $163.1 \pm 8.3$ & $64.9 \pm 11.6$ & $129.2 \pm 16.9$ & $81.3 \pm 11.3$ \\
\hline
\end{tabular}

\section{Methods}

\subsection{Patient information}

223 patients who came to the First People's Hospital of Kunshan for routine health screening agreed to participate in the present study. After a retrospective analysis of the results of health screening, 16 patients were excluded due to the presence of cardiovascular abnormalities, such as arrhythmia or severe hypertension. Among the remaining patients, 15 patients were further excluded because the measurement of oscillometric signals failed or the measured signals were unreasonably distorted in these patients. As a consequence, 192 patients were included, and they were randomly divided into two groups (namely, the 'Training' group and the 'Testing' group), with 96 patients in each group. The demographic and hemodynamic data of the patients are summarized in Table 1.

\subsection{Acquirement of in vivo data}

Oscillometric signals were measured at the left wrist of each patient using the Health Cloud Pulse Wave Instrument (Sky Innovation Technology (Shanghai) Co., Ltd, China) after the patient has rested for at least five minutes. Prior to the measurement of oscillometric signals, the instrument was first used to measure the radial arterial systolic and diastolic pressures, and then automatically switched to an oscillometric signal recording mode by fixing the operating pressure of the cuff at a constant value (being equal to the diastolic pressure of the patient). Oscillometric signal data were recorded digitally at a sampling frequency of $512 \mathrm{~Hz}$, with the recording being sustained for at least 10 cardiac cycles to guarantee the acquirement of periodic signals. The measured data were then sent to a computer via Bluetooth where the data were stored and analyzed. Immediately after the oscillometric measurement, echocardiographic measurement was performed (using GE Vivid 7, GE, USA) on each patient to determine the in vivo SV.

\subsection{Offline processing of oscillometric signals}

Wavelet analysis was applied to the oscillometric signals (or oscillometric waves) to remove high-frequency noises. Moreover, the oscillometric waves were amplified to mimic blood pressure waves in the radial artery under the cuff by calibrating its peak and valley to the measured systolic and diastolic pressures, respectively. Figure 1 shows a typical oscillometric wave obtained from the above-mentioned data processing. It is worth noting that the oscillometric device used in the study is equipped with two high-sensitive piezoelectric sensors connected to an analog digital conversion unit that operates at a sampling frequency of $512 \mathrm{~Hz}$ (can be elevated to $1000 \mathrm{~Hz}$ if necessary). The high temporal resolution of the measurement ensures that the major characteristics of the oscillometric signals are well-retained after removal of high-frequency noises via wavelet analysis. Therefore, the 


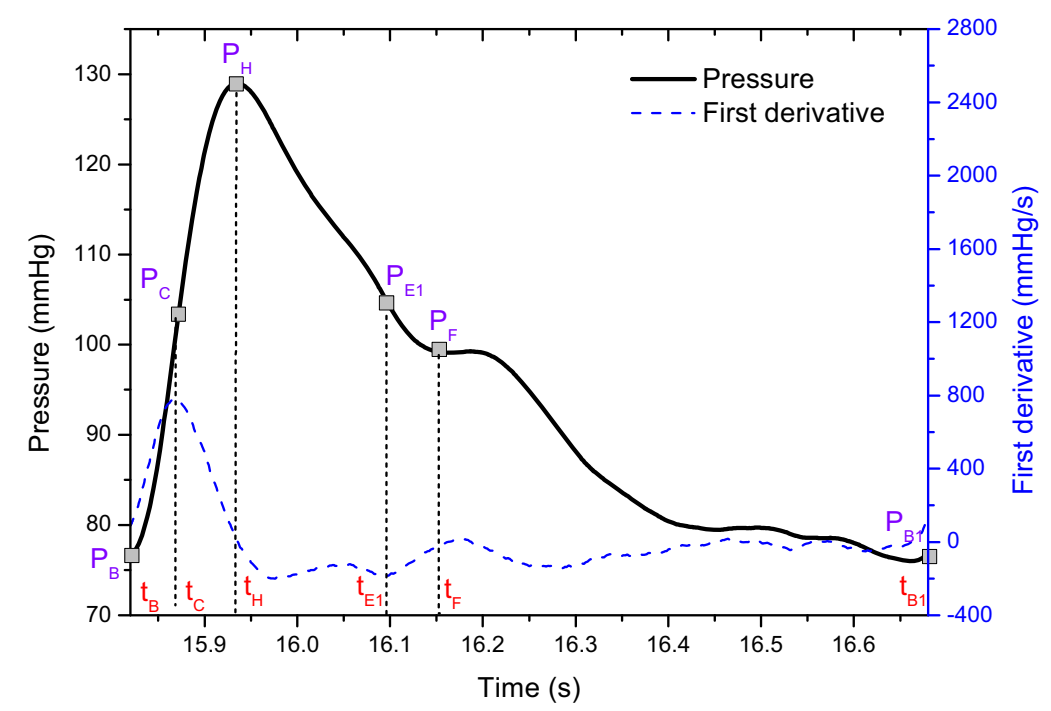

Fig. 1. A typical oscillometric wave (calibrated to blood pressures) and its first derivative. The characteristic points are denoted by $\mathrm{P}_{\mathrm{B}}, \mathrm{P}_{\mathrm{C}}, \mathrm{P}_{\mathrm{H}}, \mathrm{P}_{\mathrm{E} 1}, \mathrm{P}_{\mathrm{F}}, \mathrm{P}_{\mathrm{B} 1}$, respectively.

noise-filtering process is expected to have little influence on the selection of characteristic points. To facilitate the identification of characteristic points, the first and second derivatives of the pressure wave were calculated. $\mathrm{P}_{\mathrm{B}} / \mathrm{P}_{\mathrm{B} 1}$ and $\mathrm{P}_{\mathrm{H}}$ are the valley and peak pressure points over a cardiac duration, the identification of which is straightforward from the profile of the pressure wave. $\mathrm{P}_{\mathrm{F}}$ is the pressure point at which the first derivative of the pressure wave arrives at zero at the end of systole. $\mathrm{P}_{\mathrm{C}}$ and $\mathrm{P}_{\mathrm{E} 1}$ are the pressure points at which the second derivative of the pressure wave approaches zero prior to $\mathrm{P}_{\mathrm{H}}$ and $\mathrm{P}_{\mathrm{F}}$ points, respectively. The identified characteristic points are illustrated in Figure 1, with the times corresponding to these points denoted by $t_{B}, t_{C}, t_{H}, t_{E 1}, t_{F}$, and $t_{B 1}$, respectively.

\subsection{Method for estimating $S V$}

Our estimation method was developed on the basis of the pressure recording analytical method (PRAM) [9]. In PRAM, SV is estimated by analyzing a pressure wave measured (using photoplethysmography) at the finger using the following equation:

$$
\mathrm{SV}=\frac{A_{\mathrm{sys}}}{K \times P_{t}}
$$

where $A_{\text {sys }}$ is the area under the systolic portion of the pressure curve (see Figure 1)

$$
A_{\mathrm{sys}}=\int_{\mathrm{t}_{\mathrm{B}}}^{\mathrm{t}_{\mathrm{F}}}\left(p-\mathrm{P}_{\mathrm{B}}\right) d t,
$$

$K$ is the ratio of the expected mean arterial pressure $\left(\mathrm{P}_{\mathrm{m}, 0}\right)$ (under normal physiological conditions, it was assumed to be $90 \mathrm{mmHg}$ in [9]) to the measured mean arterial pressure $\left(\mathrm{P}_{\mathrm{m}}\right)$ 


$$
K=\frac{\mathrm{P}_{\mathrm{m}, 0}}{\mathrm{P}_{\mathrm{m}}}
$$

$P_{\mathrm{t}}$ is an analytical description of the pressure wave profile,

$$
P_{t}=\frac{\mathrm{P}_{\mathrm{H}}-\mathrm{P}_{\mathrm{B}}}{\mathrm{t}_{\mathrm{H}}-\mathrm{t}_{\mathrm{B}}}+\frac{\mathrm{P}_{\mathrm{F}}}{\mathrm{t}_{\mathrm{B} 1}-\mathrm{t}_{\mathrm{F}}}-\frac{\mathrm{P}_{\mathrm{E} 1}}{\mathrm{t}_{\mathrm{B} 1}-\mathrm{t}_{\mathrm{E} 1}} .
$$

Although Eq. (1) was found to enable a reasonable estimation of cardiac output (SV $\times$ Heart Rate) in [9], a direct application of the equation to the oscillometric waves measured in our study failed to obtain results comparable to the measured data $(r<0.03)$. A potential cause for the discrepancy is that the location of pressure wave measurement in our study (at the wrist) is different from that in [9] (at the finger), which may significantly alter the characteristics of measured pressure wave and the results of characteristic point selection. Other possible causes include differences in the principle of measurement and associated measurement errors between our study and [9]. Therefore, we took several strategies to improve the equation: 1) reformulate $P_{t}$ to utilize more information available in the measured pressure wave; 2) incorporate more patient-specific anthropometric or physiological parameters, such as height, weight and heart rate; and 3) optimize the coefficients used in the equation. The resulting equation is expressed as

$$
\mathrm{SV}=\frac{a_{\mathrm{K}} \times A_{\mathrm{sys}}}{K^{a_{\mathrm{p} \_\mathrm{K}}} \times P_{t} \times{C_{\mathrm{HR}}{ }^{{ }^{\mathrm{HR}}}}} \times C_{\mathrm{W}}{ }^{a}{ }^{\mathrm{W}} \times C_{\mathrm{HW}}{ }^{{ }^{\mathrm{HW}}}
$$

Given the fact that the exact expression of $P_{t}$ is unknown in prior, we herein included all combinations of characteristic points that are considered to possibly reflect the loading conditions of the heart. In the meantime, each item was multiplied by a coefficient to weight its contribution. The weighting coefficients were determined afterward by implementing a parameter optimization algorithm (to be detailed in the following section). The reformulated $P_{t}$ is

$$
P_{t}=a_{\mathrm{p} 11} \frac{\mathrm{P}_{\mathrm{C}}-\mathrm{P}_{\mathrm{B}}}{\mathrm{t}_{\mathrm{C}}-\mathrm{t}_{\mathrm{B}}}+a_{\mathrm{p} 12} \frac{\mathrm{P}_{\mathrm{F}}-\mathrm{P}_{\mathrm{B} 1}}{\mathrm{t}_{\mathrm{B} 1}-\mathrm{t}_{\mathrm{F}}}+a_{\mathrm{p} 13} \frac{\mathrm{P}_{\mathrm{E} 1}-\mathrm{P}_{\mathrm{B} 1}}{\mathrm{t}_{\mathrm{B} 1}-\mathrm{t}_{\mathrm{E} 1}}+a_{\mathrm{p} 14} \frac{\mathrm{P}_{\mathrm{H}}-\mathrm{P}_{\mathrm{B}}}{\mathrm{t}_{\mathrm{H}}-\mathrm{t}_{\mathrm{B}}}+a_{\mathrm{p} 15} \frac{\mathrm{P}_{\mathrm{H}}-\mathrm{P}_{\mathrm{F}}}{\mathrm{t}_{\mathrm{F}}-\mathrm{t}_{\mathrm{H}}}+a_{\mathrm{p} 16} \frac{\mathrm{P}_{\mathrm{H}}}{\mathrm{P}_{\mathrm{F}}}+a_{\mathrm{p} 17} \frac{\mathrm{P}_{\mathrm{H}}}{\mathrm{P}_{\mathrm{B} 1}}+a_{\mathrm{p} 18} \frac{\mathrm{P}_{\mathrm{H}}-\mathrm{P}_{\mathrm{B} 1}}{\mathrm{t}_{\mathrm{B} 1}-\mathrm{t}_{\mathrm{H}}}+a_{\mathrm{p} 19} \frac{\mathrm{P}_{\mathrm{H}}-\mathrm{P}_{\mathrm{C}}}{\mathrm{t}_{\mathrm{H}}-\mathrm{t}_{\mathrm{C}}},
$$

$C_{\mathrm{HR}}, C_{\mathrm{W}}$ and $C_{\mathrm{HW}}$ are new parameters incorporated to account for the effects of heart rate, weight and weight to height ratio, and they are expressed as the ratios of the measured data to the reference values 


$$
\begin{aligned}
& C_{\mathrm{HR}}=\frac{T}{T_{0}}, \\
& C_{\mathrm{W}}=\frac{W}{W_{0}}, \\
& C_{\mathrm{HW}}=\frac{W}{W_{0}} \times \frac{H_{0}}{H},
\end{aligned}
$$

where $T, W$ and $H$ represent the duration of a heartbeat, weight and height measured in each patient, respectively. Their reference values were set to be $T_{0}=1.0 \mathrm{sec}, W_{0}=70 \mathrm{Kg}$, and $H_{0}=175 \mathrm{~cm}$.

\subsection{Optimization of estimation equation}

To determine the coefficients used in Eq. (5), a parameter optimization method (herein the Nelder-Mead method [18]) was employed to search for the coefficients that give rise to a best comparison between estimated and measured SV. Herein, the data acquired in the 96 patients of the 'Training' group were used to optimize the coefficients. The optimization algorithm was implemented numerically using an in-house computer program written in FORTRAN language, and the optimization process was terminated when the root mean squared error between estimated and measured SV ceases to decrease further. A flow chart of the optimization process is illustrated in Figure 2. After coefficient optimization, the estimation equation was applied directly to the 'Testing' group to examine its validity.

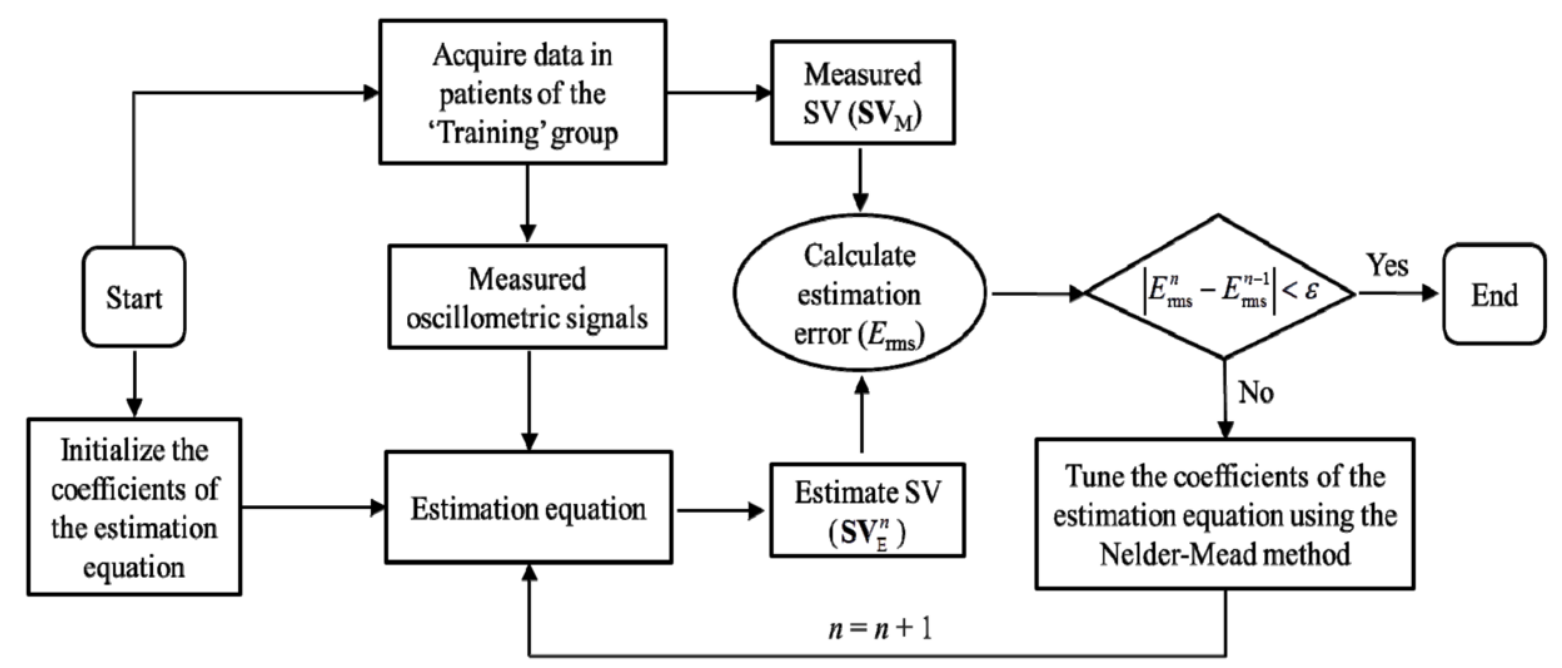

Fig. 2. Flow chart of the process for optimizing the coefficients used in Eq. (5). The estimation error $\left(E_{\mathrm{rms}}\right)$ is evaluated as the root mean square of the differences between the measured and estimated SV, $n$ represents the cycle of iteration, and $\varepsilon$ is the tolerance of convergence, herein set to be $0.01 \%$. 


\section{Results}

The estimated SV for the 'Training' group are compared with the measured data in Figure 3(a), where the estimated and measured SV are $54.82 \pm 9.33 \mathrm{ml}, 54.47 \pm 13.34 \mathrm{ml}$, respectively. A significant correlation $(\mathrm{r}=0.66, \mathrm{p}<0.0001)$ between them is observed. The corresponding Bland-Altman plot is presented in Figure 3(b), where the mean difference between the estimations and measurements is $0.36 \mathrm{ml}$, with a SD of $9.99 \mathrm{ml}$ and $95 \%$ limits of agreement from $-19.22 \mathrm{ml}$ to $19.93 \mathrm{ml}$. The 2SD-precision of the estimation expressed in percentage is $\pm 36.6 \%$. The optimized coefficient values are listed in Table 2.

Figure 4 shows the comparison between the estimated and measured SV for the 'Testing' group. Note that the estimation of SV for this group was performed by applying the estimation equation derived from the 'Training' group without further optimization of the coefficients. From Figure 4(a), a considerable correlation $(\mathrm{r}=0.60, \mathrm{p}<0.0001)$ between the estimations and measurements is confirmed. From the Bland-Altman plot (see Figure 4(b)), the mean estimation error is $-0.22 \mathrm{ml}$, with a SD of $10.71 \mathrm{ml}$ and $95 \%$ limits of agreement from $-21.22 \mathrm{ml}$ to $20.79 \mathrm{ml}$. The $2 \mathrm{SD}$-precision of the estimation expressed in percentage is $\pm 39.0 \%$.
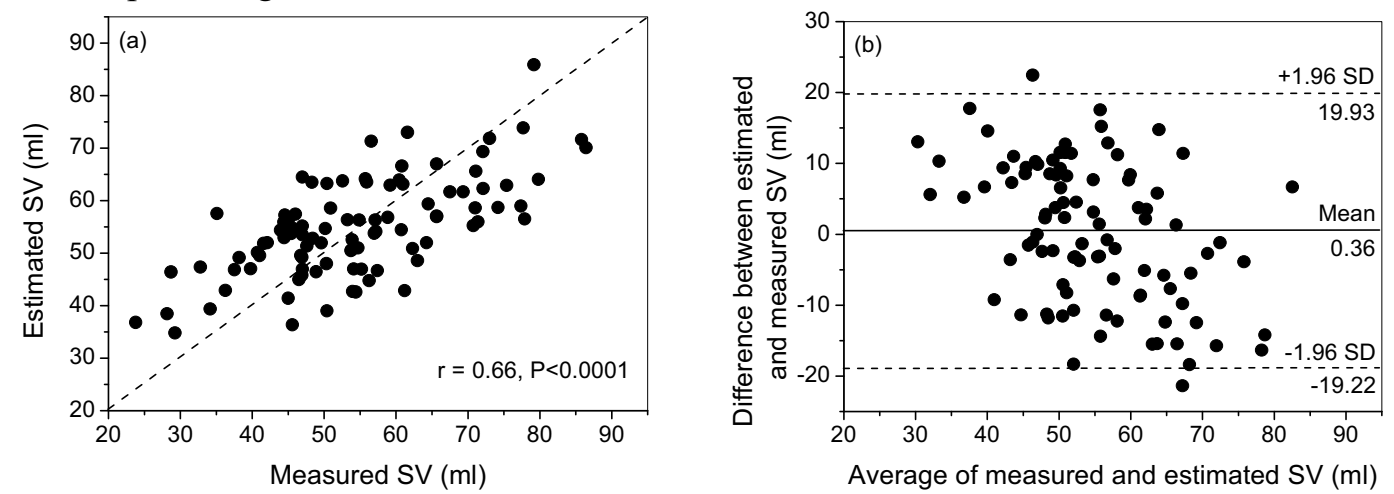

Fig. 3. Scatter plot of the estimated SV compared with the measured SV (for the 'Training' group) (a), and the corresponding Bland-Altman plot (b).
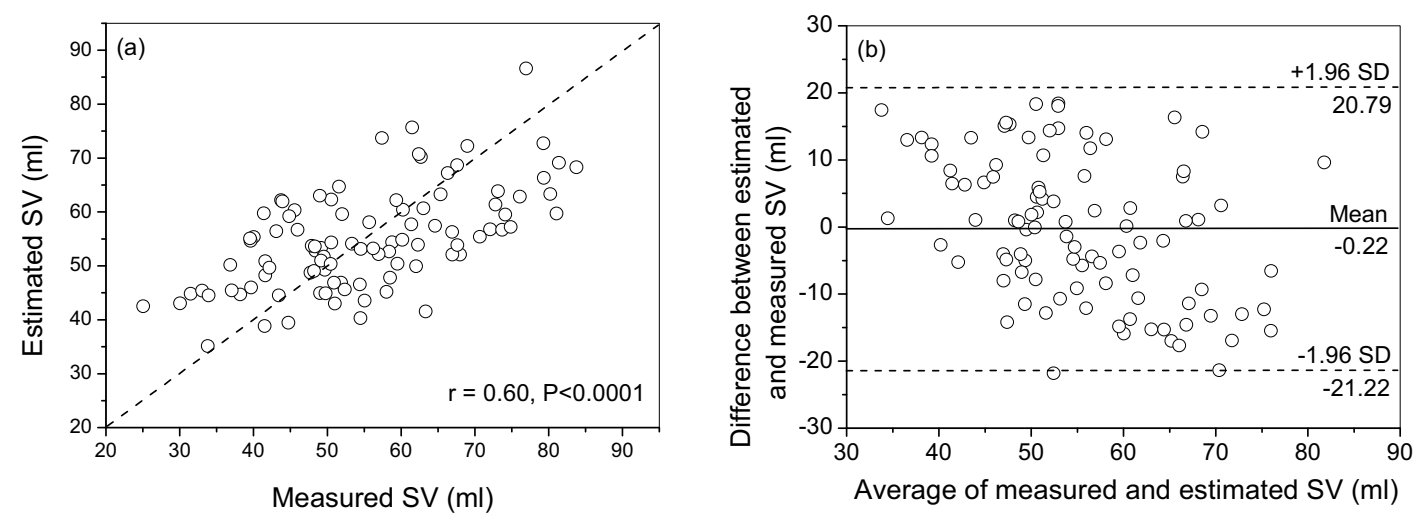

Fig. 4. Scatter plot of the estimated SV compared with the measured SV (for the 'Testing' group) (a), and the corresponding Bland-Altman plot (b). 
Table 2

Optimized values of the coefficients used in the estimation equation (Eq. (5))

\begin{tabular}{llllllll}
\hline Coefficients & $a_{\mathrm{K}}$ & $a_{\mathrm{P} \_\mathrm{K}}$ & $a_{\mathrm{p} 1}$ & $a_{\mathrm{p} 2}$ & $a_{\mathrm{p} 3}$ & $a_{\mathrm{p} 4}$ & $a_{\mathrm{p} 5}$ \\
\hline Values & 317.0 & 0.405 & -0.0828 & 1.652 & 2.40 & 0.0914 & -0.0110 \\
\hline Coefficients & $a_{\mathrm{p} 6}$ & $a_{\mathrm{p} 7}$ & $a_{\mathrm{p} 8}$ & $a_{\mathrm{p} 9}$ & $a_{\mathrm{HR}}$ & $a_{\mathrm{W}}$ & $a_{\mathrm{HW}}$ \\
\hline Values & 31.929 & -0.0331 & 1.307 & 0.0932 & 1.660 & 0.963 & -0.408 \\
\hline
\end{tabular}

\section{Discussion}

We modified the PRAM method [9] to noninvasively estimate SV from oscillometric signals recorded by a blood pressure device mounted on the wrist. The improvement of our method over the original one lies in three aspects: 1) more patient-specific anthropometric or physiological parameters were taken into account, including heart rate, height and weight; 2) the expression of the estimation equation is expanded to make adequate use of the information carried by the oscillometric waves; and 3 ) the contribution of each term in the estimation equation is weighted by a coefficient that can be determined by fitting the estimated results to the measured data. To validate our method, we divided the patients into the 'Training' group and the 'Testing' group. The clinical data of the former group were used to train the estimation equation, while the data of the latter group were used to examine the validity of the derived estimation equation. Obtained results demonstrated that the estimated SV correlated closely with the measured data for both groups with a similar range of error distribution, which implies that the estimation equation may also be applicable to patients not included in the present study. It should be stressed that although our method is intended to estimate SV, it can output $\mathrm{CO}$ as well since the oscillometric device used in our study automatically calculates the duration of each heartbeat. Furthermore, continuous monitoring of SV or CO with the method is feasible since SV is estimated on a beat-to-beat basis. Methodologically, the ways in which we design and optimize the estimation equation may also have application in studies dedicated to the development of similar methods. For instance, the original PRAM method has been found to considerably underestimate SV in morbidly obese patients [19]. Our method may be applied to construct a new estimation equation suitable for this patient cohort.

With respect to the precision of estimation, our method (2SD-precision being $\pm 36.6 \% \sim \pm 39.0 \%$ ) is comparable to some widely-used commercial devices (for instance, noncalibrated Modelflow $( \pm 31 \%)$, PiCCOplus $( \pm 32 \%)$, and Flotrac-Vigileo $( \pm 41 \%)$ ) [15], but is inferior to other devices, especially those with invasive measurement-based calibration, such as, calibrated-Modelflow $( \pm 17 \%)$, LiDCOplus $( \pm 24 \%)$ [15]. Similar to other pulse wave analysis-based methods, our method is vulnerable to sources of errors related to the quality of pressure wave measurement. In particular, the employed oscillometric method for measuring peripheral pressure waves is, in nature, more susceptible to external perturbations, which may introduce uncertain errors in the estimation. This problem may be partially solved by repeated measurements, although the issue has not been addressed in the present study due to limitations associated with the data acquirement system. Another problem that may compromise the precision of the estimation is that oscillometric signals and echocardiographic SV have been taken in sequence rather than simultaneously in the present study. Since SV varies with heart rate which is highly sensitive to normal fluctuations in physiological conditions, the echocardiographic SV may deviate from the in vivo SV at the moment of oscillometric measurement. Moreover, the majority of the patients involved in our study are middle-aged, which raises a question 
as to whether the estimation equation is applicable to younger or older subjects. Finally, it is worth noting that the method has been developed based on oscillometric signals measured under resting conditions, whether it is applicable to signals measured under other physiological conditions, such as during mild to moderate exercise, remains to be investigated. We are now working together with the clinical staffs to refine the experimental protocol such that these limitations can be addressed in our future studies.

Despite the above-mentioned limitations, this study is a valuable attempt to investigate the feasibility of deriving SV or CO from oscillometric signals measured in the peripheries. The fully noninvasive nature of the method and the ease of oscillometric measurement make it possible to estimate SV or $\mathrm{CO}$ in a home-based environment. In particular, the method can be applied in combination with a cloud data management system which continuously stores the results of measurement, such that the data of each patient can be tracked, reviewed and analyzed over a long period. This opens the possibility of investigating the significance of continuous SV or CO monitoring in assessing the risk of cardiovascular events or performing pharmacological studies to evaluate the effectiveness of certain medications in improving cardiovascular function.

\section{Conclusion}

A new method has been developed to estimate SV from oscillometric signals measured at the wrist using an automatic blood pressure device. The estimation equation was first optimized based on the clinical data acquired from the 'Training' patient group, followed by a testing procedure performed on the 'Testing' patient group. For both groups, a significant correlation between the estimated results and measured data was confirmed. Advantaged by the fully noninvasive and calibration-free characteristics, our method is suitable for use in a home-based environment, although further studies would be needed to reduce uncertain errors associated with oscillometric measurement and to examine the method in a larger population with a wider age distribution.

\section{Acknowledgement}

Zhipeng Deng was supported by the Hui-Chun Chin and Tsung-Dao Lee Chinese Undergraduate Research Endowment; Fuyou Liang was supported by the National Natural Science Foundation of China (Grant No. 81370438) and the SJTU Medical-Engineering Cross-cutting Research Foundation (Grant No. YG2012MS24). Special thanks to Mr. Kaiwen Ren for his technical support.

\section{References}

[1] A.M. de Wolf, Perioperative assessment of the cardiovascular system in ESLD and transplantation, Int. Anesthesiol. Clin. 44 (2006), 59-78.

[2] G. de Simone, R.B. Devereux, A. Ganau, R.T. Hahn, P.S. Saba, G.F. Mureddu, M.J. Roman and B.V. Howard, Estimation of left ventricular chamber and stroke volume by limited M-mode echocardiography and validation by two-dimensional and Doppler echocardiography, The American Journal of Cardiology 78 (1996), 801-807.

[3] R.M. Lang, M. Bierig, R.B. Devereux, F.A. Flachskampf et al., Recommendations for chamber quantification, Eur. J. Echocardiogr. 7 (2006), 79-108.

[4] S. Jhanji, J. Dawson and R.M. Pearse, Cardiac output monitoring: Basic science and clinical application, Anaesthesia 63 (2008), 172-181. 
[5] S. Harvey, D.A. Harrison, M. Singer et al., Assessment of the clinical effectiveness of pulmonary artery catheters in management of patients in intensive care (PAC-Man): A randomised controlled trial, Lancet 366 (2005), 472-477.

[6] K.H. Wesseling, J.R. Jansen, J.J. Settels and J.J. Schreuder, Computation of aortic flow from pressure in humans using a nonlinear, three-element model, J. Appl. Physiol. 74 (1993), 2566-2573.

[7] L.W. Bogert, K.H. Wesseling, O. Schraa, E.J. Van Lieshout, B.A. de Mol, J. van Goudoever, B.E. Westerhof and J.J. van Lieshout, Pulse contour cardiac output derived from non-invasive arterial pressure in cardiovascular disease, Anaesthesia 65 (2011), 1119-1125.

[8] R. Mukkamala, A.T. Reisner, H.M. Hojman, R.G. Mark and R.J. Cohen, Continuous cardiac output monitoring by peripheral blood pressure waveform analysis, IEEE Trans. Biomed. Eng. 53 (2006), 459-467.

[9] S.M. Romano and M. Pistolesi, Assessment of cardiac output from systemic arterial pressure in humans, Crit. Care Med. 30 (2002), 1834-1841.

[10] G. Biancofiore, L.A. Critchley, A. Lee et al., Evaluation of an uncalibrated arterial pulse contour cardiac output monitoring system in cirrhotic patients undergoing liver surgery, Br. J. Anaesth. 102 (2009), 47-54.

[11] K. Bendjelid, G. Marx, N. Kiefer, T.P. Simon, M. Geisen, A. Hoeft, N. Siegenthaler and C.K. Hofer, Performance of a new pulse contour method for continuous cardiac output monitoring: Validation in critically ill patients, Br. J. Anaesth. 111 (2013), 573-579.

[12] M. Calamandrei, L. Mirabile, S. Muschetta, G.F. Gensini, L. De Simone and S.M. Romano, Assessment of cardiac output in children: A comparison between the pressure recording analytical method and Doppler echocardiography, Pediatr. Crit. Care. Med. 9 (2008), 310-312.

[13] P. Giomarelli, B. Biagioli and S. Scolletta, Cardiac output monitoring by pressure recording analytical method in cardiac surgery, Eur. J. Cardiothorac. Surg. 26 (2004), 515-520.

[14] T.W. Felbinger, D.A. Reuter, H.K. Eltzschig, K. Moerstedt, O. Goedje and A.E. Goetz, Comparison of pulmonary arterial thermodilution and arterial pulse contour analysis: Evaluation of a new algorithm, J. Clin Anesth. 14 (2002), 296-301.

[15] B.F. Geerts, L.P. Aarts and J.R. Jansen, Methods in pharmacology: Measurement of cardiac output, Br. J. Clin. Pharmaco. 171 (2011), 316-330.

[16] F.Y. Liang, S. Takagi, R. Himeno and H. Liu, A computational model of the cardiovascular system coupled with an upper-arm oscillometric cuff and its application to studying the suprasystolic cuff oscillation wave concerning its value in assessing arterial stiffness, Comput. Methods Biomech. Biomed. Engin. 16 (2013), 141-157.

[17] J. Baulmann, U. Schillings, S. Rickert, S. Uen, R. Düsing, M. Illyes, A. Cziraki, G. Nickering and T. Mengden, A new oscillometric method for assessment of arterial stiffness: Comparison with tonometric and piezo-electronic methods, J. Hypertens. 26 (2008), 523-528.

[18] F.Y. Liang, K. Sughimoto, K. Matsuo, H. Liu and S. Takagi, Patient-specific assessment of cardiovascular function by combination of clinical data and computational model with applications to patients undergoing fontan operation, Int. J. Numer. Method Biomed. Eng. (In press).

[19] D.P. Bernstein, Pressure pulse contour-derived stroke volume and cardiac output in the morbidly obese patient, Obes. Surg. 18 (2008), 1015-1021. 